\title{
Diferencias individuales en la impulsividad y la flexibilidad cognitiva en adultos jóvenes sanos*
}

\author{
Individual differences in impulsivity and cognitive flexibility in \\ healthy young adults
}

Diferenças individuais na impulsividade e flexibilidade cognitiva
em adultos jovens saudáveis Jorge Emiro Restrepo $0^{* * *}$
Juan David Soto******an
Alejandro Rivera*****.

Recibido 28. 02. 2016 • Arbitrado 26. 03. 2016 • Aprobado 13. 04. 2016

\section{Resumen}

Introducción: Las causas de la impulsividad no se ha establecido aún y coexisten diversos modelos teóricos que incluyen diferentes factores. Varias investigaciones han analizado la relación entre el funcionamiento neuropsicológico y la impulsividad. La relación entre la personalidad y la flexibilidad cognitiva tampoco ha sido analizada en profundidad. La Neurociencia de la Personalidad, disciplina recientemente establecida, se ha concentrado en analizar los correlatos cognitivos de los factores de la personalidad. Objetivo: analizar las correlaciones entre la personalidad sana, la flexibilidad cognitiva y la impulsividad. Me-

Este artículo es producto de la investigación "Factores de personalidad, flexibilidad cognitiva e impulsividad en adultos jóvenes sanos" que se realizó con financiación de la Universidad Cooperativa de Colombia-Medellín y del Comité Nacional de Investigación Codei de la misma universidad, año 2014-2015.

** Biólogo, Universidad de Antioquia, Medellín; Psicólogo, Universidad Cooperativa de Colombia-Medellín, Colombia; Doctor y Máster en Neuropsicología, Universidad de Salamanca, España; Profesor Tiempo Completo, Facultad de Psicología, Universidad Cooperativa de Colombia, Sede Medellín, Colombia, jemiror@gmail.com

**** Psicólogo, Universidad Cooperativa de Colombia-Medellín, Colombia; Magister en Neuropsicología Universidad de San Buenaventura, Medellín, Colombia; Profesor Tiempo Completo, Facultad de Psicología, Universidad Cooperativa de Colombia, Sede Medellín, Colombia, juan.soto@campusucc.edu.co

Psicólogo, Universidad Cooperativa de Colombia-Medellín, Colombia, rivera_ortiz_alejandro@hotmail.com 
todología: se trabajó con una muestra de 80 estudiantes universitarios de pregrado sin antecedentes de trastorno psicológico o neurológico, sin antecedentes familiares de trastorno psicológico o neurológico, y sin antecedentes de consumo de sustancias psicoactivas en los últimos seis meses. Se utilizaron el Inventario de Personalidad NEO Revisado, la Escala de Impulsividad de Barrat (BIS-11), el TMT-B y el Test de Clasificación de Tarjetas de Wisconsin (WCST). Resultados: el análisis de correlaciones mostró que la impulsividad, en relación con los factores de la personalidad, solo presentó una correlación estadísticamente significativa $(\mathrm{p}<$.05) con la Amabilidad. Respecto a la relación entre la impulsividad y las facetas de la personalidad, solo la Estética y la Franqueza presentaron correlaciones estadísticamente significativas $(\mathrm{p}<$.05). Conclusiones: solo el factor Amabilidad presentó una correlación estadísticamente significativa con la impulsividad. Este hallazgo no solo coincide con lo reportado en la literatura sobre neurociencia de la personalidad sino que es plenamente consistente con los atributos cognitivos que deberían estar asociados con el comportamiento que exhiben este tipo de personas.

Palabras clave: personalidad, diferencias individuales, cognición, neurociencias.

\section{Abstract}

Introduction: The cause of impulsivity has not been established yet and coexist various theoretical models that include different factors. Several studies have examined the relationship between neuropsychological functioning and impulsivity. The relationship between personality and cognitive flexibility has not been analyzed in depth. The Neuroscience of Personality, newly established discipline, has focused on analyzing the cognitive correlates of personality factors. Objective: To analyze correlations between healthy personality, cognitive flexibility and impulsivity. Methodology: we worked with a sample of 80 undergraduate students with no history of psychological or neurological disorder, no family history of psychological or neurological disorder, 
and no history of substance use in the past six months. The Revised NEO Personality Inventory, the Barratt Impulsiveness Scale (BIS- 11), the TMT- B and Test Wisconsin Card Sorting (WCST) were used. Results: The correlation analysis showed that impulsivity in relation to personality factors, only a statistically significant correlation $(\mathrm{p}<.05)$ with Kindness. Regarding the relationship between impulsivity and personality facets, only Aesthetics and Openness showed statistically significant correlations $(\mathrm{p}<$.05). Conclusions: Friendliness factor only had a statistically significant correlation with impulsivity. This finding not only coincides with that reported in the literature on personality neuroscience but is fully consistent with the cognitive attributes that should be associated with the behavior that exhibit this type of person.

Key words: personality, individual differences, cognition, neurosciences.

\section{Resumo}

Introdução: As causas da impulsividade ainda não foi estabelecida e coexistem vários modelos teóricos que incluem fatores diferentes. Vários estudos analisaram a relação entre o funcionamento neuropsicológico e impulsividade. A relação entre personalidade e flexibilidade cognitiva não foi analisado em profundidade. Personalidade Neuroscience, disciplina recentemente criada, com foco na análise dos correlatos cognitivos de fatores de personalidade. Objetivo: Analisar as correlações entre personalidade saudável, flexibilidade cognitiva e impulsividade. Metodologia: trabalhou com uma amostra de 80 alunos de graduação, sem histórico de distúrbio psicológico ou neurológico, sem história familiar de distúrbio psicológico ou neurológico, e sem histórico de uso de drogas nos últimos seis meses. o Inventário de Personalidade NEO Revisado, foram utilizados a escala de impulsividade de Barrat (BIS-11), a TMT-B e Teste de Wisconsin (WCST). Resultados: A análise de correlação mostrou que impulsividade, em relação aos fatores de personalidade, apenas uma correlação estatisticamente significativa $(\mathrm{p}<0,05)$ com bondade. Quanto à relação entre impulsividade e de personalidade facetas, apenas a estética e franqueza eles 
apresentaram correlação estatisticamente significativa ( $\mathrm{p}<0,05)$. Conclusões: Simpatia único fator mostrou uma correlação estatisticamente significativa com a impulsividade. Esta descoberta não só coincide com o relatado na literatura sobre a personalidade neurociência, mas é totalmente consistente com atributos cognitivos que devem ser associados ao comportamento exibido essas pessoas.

Palavras-chave: personalidade, diferenças individuais, cognição, neurociência.

\section{Introducción}

La impulsividad ha sido una de las características (rasgos o estilos) psicológicos de mayor relevancia teórica dentro de las diversas disciplinas que se ocupan del estudio de los procesos mentales, emocionales y del comportamiento (Squillace, Picón \& Schmidt, 2011). Igualmente, es una característica fundamental para las conceptualizaciones en torno a la personalidad (DeYoung, 2010b) y ocupa un lugar nuclear dentro de las explicaciones en psicopatología (Vaz-Leal et al., 2014). También es un factor determinante para comprender múltiples temas en psicología social (Strack \& Deutsch, 2004). El estudio de la impulsividad, el control del comportamiento y la capacidad de retrasar la gratificación son aspectos esenciales en el marco programático de una psicología de las diferencias individuales interesada en contribuir a la salud y al bienestar general de las personas y los grupos (Cyders \& Coskunpinar, 2011; Kisa, Yildirim \& Göka, 2005).

La impulsividad se ha asociado a diferentes tipos de trastornos psicopatológicos, como la ansiedad (Perugi et al., 2011) la depresión y la manía (Swann, Steinberg, Lijffijt \& Moeller, 2008), la bulimia (Fischer, Smith \& Anderson, 2003), el déficit de atención (Kenemans et al., 2005), la adicción al alcohol (Lawrence, Luty, Bogdan, Sahakian \& Clark, 2005), el trastorno límite de la personalidad (Berlin, Rolls \& Iversen, 2005) y el trastorno antisocial de la personalidad (Alcorn et al., 2013). La incapacidad para regular el comportamiento y la presencia de conductas reflejas motivadas por la gratificación inmediata 
son, igualmente, un problema cotidiano en el contexto de la psicología infantil (Hinshaw, 2003).

La impulsividad se ha entendido como una dimensión en cuyo extremo contrario se sitúa la reflexividad (Zern, Kenney \& Kvaraceus, 1977). Como concepto teórico, la impulsividad es un constructo multifactorial que incluye la tendencia a actuar irreflexivamente, por "impulso", con poca o ninguna consideración por las consecuencias; la presencia de comportamientos poco regulados y organizados, caracterizados por ser de aparición refleja y no deliberada; y la falta de consciencia sobre las acciones y sus efectos (Lassiter, 2009; Madden \& Bickel, 2010). En general, la impulsividad se caracteriza por la ausencia de deliberación sobre las acciones, la tendencia refleja o reactiva de los comportamientos y la incapacidad para contener los actos cuando existe una gratificación inmediata (Lassiter, 2009; Madden \& Bickel, 2010).

Las causas de la impulsividad no se ha establecido aún y coexisten diversos modelos teóricos que incluyen diferentes factores. Algunos de los modelos más referenciados actualmente incluyen factores neuropsicológicos, neuroquímicos y de personalidad dentro del circuito causal del control de los impulsos (Pickering, 2004). La flexibilidad cognitiva, el control atencional, la memoria de trabajo y, en general, varias de las funciones ejecutivas se han asociado neuropsicológicamente con la impulsividad (Kelly, Bulik \& Mazzeo, 2013; Wing, Rabin, Wass \& George, 2013). Los niveles de dopamina y serotonina en ciertas regiones de la corteza orbitofrontal también se han relacionado con el mecanismo neuroquímico implicado en la impulsividad (Dalley \& Roiser, 2012). Finalmente, ciertos rasgos y factores de la personalidad, como la búsqueda de sensaciones, se han correlacionado con la impulsividad (Hur \& Bouchard, 1997).

Varias investigaciones han analizado la relación entre el funcionamiento neuropsicológico y la impulsividad (Pickering, 2004; Alcázar-Córcoles, Verdejo-García, Bouso-Saiz \& Bezos-Saldaña, 2010). La impulsividad se asocia con la incapacidad para contener o controlar ciertos tipos de comportamientos inadecuados o con cierta aversión 
a la espera. Se ha sugerido que esta incapacidad puede estar causada por una deficiencia de control inhibitorio (Roberts, Fillmore \& Milich, 2011; Logan, Schachar \& Tannock, 1997). Aunque teóricamente parece plausible, no todos los resultados han sido positivos. Sin embargo, sí existe evidencia suficiente para considerar la sintomatología asociada a la impulsividad como una variación en el funcionamiento de los mecanismos de control asociados con la corteza prefrontal, en particular en la corteza lateral prefrontal y la corteza cingulada anterior (Torregrossa, Quinn \& Taylor, 2008).

Aunque la falta de control de impulsos asociada con la impulsividad es una de las características principales de los traumatismos craneoencefálicos donde existe compromiso frontal (Yurgelun-Todd et al., 2005; Votruba et al., 2008), no necesariamente debe considerarse la impulsividad como sinónimo de alteración debida a la pérdida de una función. El control de los impulsos puede ser conceptuado como un constructo dimensional con extremos de "exceso" y "defecto". Ambos casos podrían considerarse como una variación de la función, entendiendo ésta como concepto normativo. Es decir, el funcionamiento normal del control de los impulsos debería estar en el punto intermedio de la dimensión, y las variaciones en la dimensión, bien sean positivas o negativas, representarían un caso de disfunción, alteración o variación.

Este último concepto, el de variación, se utiliza más en el contexto de la Psicología de las Diferencias Individuales (Chamorro-Premuzic, 2011; Mahoney, 2011). Esta vertiente teórica de la Psicología General asume que los procesos cognitivos, emocionales y comportamentales pueden variar cuantitativamente a lo largo de una dimensión cuyo eje central equivaldría al punto óptimo o nivel normativo deseado. Todas las variaciones de los diferentes procesos contribuirían al establecimiento de las diferencias individuales que constituyen la personalidad. De aquí que ambos conceptos, "personalidad" y "diferencias individua- 
les”, estén tan estrechamente vinculados (Chamorro-Premuzic, 2011; Mahoney, 2011).

Como el propósito de la Psicología de las Diferencias Individuales es establecer las variaciones en los diferentes procesos cognitivos, emocionales y comportamentales, es usual que los estudios se desarrollen con personas sanas y no con muestras clínicas, como ocurre en la neuropsicología clínica tradicional o los estudios en psicopatología. Esta aclaración es importante hacerla porque no es común que las pruebas e instrumentos de evaluación neuropsicológica sean aplicados en población sana. Por esta razón no es muy alto el número de investigaciones en esta área.

Tradicionalmente se ha asociado la impulsividad con el factor Extraversión (Campbell \& Heller, 1987; Eysenck \& Eysenck, 1978; Plomin, 1976). Debido a las características cognitivas y comportamentales de las facetas de la extraversión, la impulsividad se ha mostrado como un componente asociado del rasgo (Karin, Helmers, Young \& Pihl, 1997). Sin embargo, el nivel de correlación varían en función del instrumento que se utilice para evaluar la impulsividad, así como de la forma como se haya conceptuado el término. Lo mismo ocurre con la relación con el factor Neuroticismo, aunque esta asociación es más estable debido a que la impulsividad es, en sí misma, una faceta del factor. La correlación entre la impulsividad y la Amabilidad se ha reportado como negativa (Laursen, Pulkkinen \& Adams, 2002). La asociación entre la Responsabilidad y la impulsividad no se ha reportado pero puede suponerse que es negativa, debido a las características del factor. La relación entre la Apertura a la experiencia y la impulsividad tampoco no se ha reportado.

La relación entre la personalidad y la flexibilidad cognitiva tampoco ha sido analizada en profundidad. La Neurociencia de la Personalidad (DeYoung, 2010b; DeYoung \& Gray, 2009), disciplina recientemente establecida, se ha concentrado en analizar los correlatos cognitivos de los factores de la personalidad. El Neuroticismo, en particular, está asociado con la rumiación 
cognitiva que es, por definición, una forma de inflexibilidad mental (Flehmig, Steinborn, Langner \& Westhoff, 2007). La Apertura a la experiencia (Ritter et al., 2012), la Responsabilidad (Moore \& Malinowski, 2009) y la Extraversión (Su, Chung $\& \mathrm{Su}, 2012)$, por el contrario, se han asociado con altos niveles de flexibilidad cognitiva.

La relación entre la flexibilidad cognitiva y la impulsividad se ha analizado en algunas psicopatologías, como en el trastorno obsesivo compulsivo y la tricotilomanía (Chamberlain, Fineberg, Blackwell, Robbins \& Sahakian, 2006), o en los trastornos alimentarios (Galimberti, Martoni, Cavallini, Erzegovesi \& Bellodi, 2012), o personas alcohólicas (Semenova, 2012). De igual manera, esta asociación no se analizado en profundidad en personas sanas y la mayoría de los estudios se han concentrado en analizar las relaciones entre ambas variables en población con algún tipo de psicopatología o alteración neurológica o neuropsicológica.

En conclusión, las relaciones entre la personalidad, la flexibilidad cognitiva y la impulsividad se han analizado en población clínica con diferentes psicopatologías, alteraciones neuropsicológicas o afecciones neurológicas, pero los estudios con población sana apenas comienzan. La neurociencia de las diferencias individuales es un área de inicio reciente y esta investigación, que pretende analizar las correlaciones entre la personalidad sana, la flexibilidad cognitiva y la impulsividad, es una contribución empírica que, en castellano, representa uno de los pocos estudios realizados en el campo.

\section{Metodología \\ Muestra}

Estuvo constituida por 80 estudiantes universitarios de la carrera de psicología de una universidad privada de la ciudad de Medellín. Los estudiantes fueron seleccionados con base en los siguientes criterios: a) estado mental clínicamente normal establecido con 28 puntos o más en el Mini Mental State Examination, b) edad entre 18 y 25 años, c) sin antecedentes de trastorno psicológico o neurológico, d) sin antecedentes familiares de trastorno psicológico o neurológico, e) sin consumo 
de sustancias psicoactivas, incluyendo psicofármacos, en los seis últimos meses. El 70\% fueron mujeres. La edad promedio fue de 22 años.

\section{Instrumentos}

Para la evaluación de la personalidad se utilizó el NEO-PI-R. El Inventario de Personalidad NEO Revisado (Costa \& McCrae, 1992) es un autoinforme de 240 ítems en escala tipo Likert de cinco opciones de respuesta diseñado para evaluar los cinco grandes factores de la personalidad, cada factor evaluado mediante 48 ítems, y seis facetas por cada factor, evaluadas mediante 30 escalas específicas de 8 ítems cada una. El inventario ha demostrado tener unas propiedades psicométricas adecuadas (Church \& Katigbak, 2002;, McCrae \& Costa, 2010; McCrae \& Costa, 1983) y es uno de los instrumentos más utilizados para la evaluación de la personalidad normal.

Para la evaluación de la impulsividad se utilizó la Escala de Impulsividad de Barrat (BIS-11) (Barratt \& Patton, 1983). Es un autoreporte de 30 ítems elaborado para valorar los niveles de impulsividad en tres dimensiones: cognitiva (tendencia a tomar decisiones rápidas), motora (tendencia a actuar de forma súbita) y ausencia de planificación (mayor interés por el presente que por el futuro), con opciones de respuesta de frecuencia (nunca o raramente, de vez en cuando, a menudo y siempre o casi siempre) y puntuación de 1 a 4 . El BIS-11 ha demostrado tener unas propiedades psicométricas adecuadas (Fossati, Di Ceglie, Acquarini \& Barratt, 2001; Hartmann, Rief \& Hilbert, 2001) y es uno de los instrumentos más utilizados para la evaluación de impulsividad en contextos clínicos y de investigación.

Para la evaluación de la flexibilidad cognitiva se utilizaron el Test de Clasificación de Tarjetas de Wisconsin (WCST) y el Test de Apertura de Caminos parte B (TMT-B). El WCST (Heaton, Chelune, Talley, Kay \& Curtis, 1993) es una prueba neuropsicológica tradicionalmente utilizada en evaluación clínica para establecer la funcionalidad de los procesos cognitivos asociados con el razonamiento abstracto, la formación de conceptos y la generación de estrategias para afrontar contingencias 
(Eling, Derckx \& Maes, 2008). La prueba está configurada a partir de 128 tarjetas estímulo que varían en la forma (triángulo, cuadrado, redonda o cruz), el color (rojo, azul, verde o amarillo) y el número (uno, dos, tres o cuatro) y que son presentadas de forma que el evaluado debe inferir la asociación de una tarjeta en relación con cuatro tarjetas referentes. El criterio de emparejamiento entre las tarjetas varía cada cierto número de ensayos y la persona debe ajustar su decisión con base en la comprensión de la lógica de la prueba.

El TMT-B (Reitan, 1959) es una prueba neuropsicológica utilizada para evaluar, entre otras funciones cognitivas, la flexibilidad y la rapidez mental (Tombaugh, 2004). El test está incluido dentro de los protocolos y baterías de evaluación neuropsicológica más importantes (Lezak, 1995). Es una prueba de papel y lápiz. En una hoja aparecen dibujados, entre círculos, los números del 1 al 13 y las letras de la "A" a la "L". El evaluado debe unir, lo más rápido que le sea posible y sin levantar la mano del papel, los círculos en orden, comenzando por el número uno e intercalando letras y números (1, A, 2, B, etc.).

\section{Procedimiento}

Los estudiantes fueron invitados a participar en la investigación de forma voluntaria. Se reunió un grupo inicial de 100 estudiantes, pero 15 fueron excluidos con base en los criterios. Otros cuatro participantes decidieron retirarse voluntariamente y uno fue excluido por presentar altos niveles de ansiedad ante las pruebas. No se ofreció ningún tipo de retribución por la participación. El inventario de personalidad y la escala de impulsividad fueron administrados de forma individual. Cada participante recibió la instrucción necesaria para su diligenciamiento. Las pruebas neuropsicológicas fueron administradas por un neuropsicólogo a cada uno de los participantes en una cabina de evaluación psicológica de un laboratorio de psicología. Los datos fueron registrados y analizados en SPSS v. 21. 


\section{Análisis}

Se realizaron los análisis descriptivos. Se obtuvieron los valores de las medias, las desviaciones típicas y las varianzas para todas las variables. Se realizó la prueba de Kolmogorov-Smirnov con la modificación de Lillierfors para evaluar la normalidad de los datos y el Test de Levene para evaluar la homogeneidad de las varianzas de los datos. Ninguna de las variables cumplió con las dos condiciones para ser analizada de forma paramétrica. Por tal razón, los análisis fueron no paramétricos. Se realizó, entonces, un análisis de correlaciones lineal bivariado mediante el coeficiente Spearman. También se realizó la prueba t de student para analizar la varianza de las medias entre ambos sexos.

\section{Resultados}

No hubo diferencias estadísticamente significativas detectadas por la t de student en los análisis de varianza de las medias entre los sexos para ninguna de las variables. En la tabla 1 se presentan los estadísticos descriptivos para todas las variables y los resultados de los análisis de varianza de las medias. Se presentan la media, la desviación típica y la varianza para los cinco factores de la personalidad y para las seis facetas de cada uno de los cinco factores. También están los mismos estadísticos para la impulsividad, el TMTB y para las seis variables que se tuvieron en consideración del WCST. Éstas variables fueron: número de aciertos, porcentaje de errores perseverativos, porcentaje de errores no perseverativos, porcentaje de respuestas conceptuales y número de categorías.

En la tabla 2 se presentan los análisis de las correlaciones entre las variables de personalidad, de flexibilidad cognitiva y de impulsividad. El análisis de correlaciones mostró que la impulsividad, en relación con los factores de la personalidad, solo presentó una correlación estadísticamente significativa $(p<.05)$ con la Amabilidad. Respecto a la relación entre la impulsividad y las facetas de la personalidad, solo la Estética (Apertura a la experiencia) y la Franqueza (Amabilidad) presentaron correlaciones estadísticamente significativas $(p<.05)$. 
La impulsividad no presentó ninguna correlación estadísticamente significativa con ninguna de las variables de flexibilidad cognitiva. Sin embargo, el valor de la correlación entre la impulsividad y el test TMT-B fue relevante tanto por su valor como por el signo de la correlación (tabla 2). Además, el TMT-B correlacionó significativamente ( $p$ $<.05$ y $p<.01)$ con todas las variables del WCST, lo cual es evidencia de una posible asociación indirecta entre la impulsividad y el WCST.

Respecto a las correlaciones entre la flexibilidad cognitiva y las variables de personalidad, el TMT-B correlacionó significativamente ( $p$ $<$.05) con las facetas "Franqueza" y "Modestia" del factor Amabilidad. De las variables consideradas del WCST, el número de aciertos correlacionó con la faceta Vulnerabilidad del factor Neuroticismo. La variable "Errores perseverativos" correlacionó significativamente con el factor Neuroticismo $(p<.01)$ y con sus facetas de "Depresión" $(p<.01)$, "Hostilidad" ( $p<.05)$, "Vulnerabilidad" e "Impulsividad" $(p<.05)$.

La variable "Errores no perseverativos" correlacionó significativamente $(p<$.05) con las facetas "Ansiedad social" y "Vulnerabilidad" del factor Neuroticismo. La variable "Respuestas conceptuales" no correlacionó significativamente con ninguna de las variables de personalidad. La variable "Categorías" correlacionó significativamente con el factor de Neuroticismo $(p<.01)$ y con sus facetas de "Hostilidad" $(p<.01)$ y "Depresión" $(p<.05)$. La variable "Tiempo" solo correlacionó significativamente $(p<.05)$ con la faceta "Competencia" del factor Responsabilidad. 
Tabla 1. Estadísticos descriptivos de las variables de personalidad, flexibilidad cognitiva e impulsividad.

\begin{tabular}{|c|c|c|c|}
\hline & Media & Desv. Típ. & Varianza \\
\hline \multicolumn{4}{|c|}{ Factores de la personalidad } \\
\hline Neuroticismo & 92,80 & 19,42 & 377,49 \\
\hline Extraversión & 113,90 & 19,70 & 388,20 \\
\hline Apertura & 115,44 & 18,08 & 327,07 \\
\hline Amabilidad & 108,67 & 15,82 & 250,53 \\
\hline Responsabilidad & 117,15 & 19,44 & 377,97 \\
\hline \multicolumn{4}{|c|}{ Facetas del Neuroticismo } \\
\hline NAnsiedad & 18,73 & 4,77 & 22,82 \\
\hline NHostilidad & 14,26 & 4,17 & 17,41 \\
\hline NDepresión & 14,40 & 5,84 & 34,20 \\
\hline NAnsiedadSocial & 14,86 & 4,00 & 16,04 \\
\hline NImpulsividad & 16,92 & 4,13 & 17,13 \\
\hline NVulnerabilidad & 13,61 & 4,17 & 17,45 \\
\hline \multicolumn{4}{|c|}{ Facetas de la Extraversión } \\
\hline ECordialidad & 21,51 & 3,92 & 15,43 \\
\hline EGregarismo & 16,38 & 6,21 & 38,63 \\
\hline EAsertividad & 18,03 & 5,33 & 28,46 \\
\hline EActividad & 17,57 & 4,45 & 19,81 \\
\hline EBusqEmocion & 18,51 & 4,53 & 20,60 \\
\hline EEmocPosit & 21,98 & 5,06 & 25,66 \\
\hline \multicolumn{4}{|c|}{ Facetas de la Apertura } \\
\hline ApFantasia & 20,42 & 5,77 & 33,30 \\
\hline ApEstetica & 19,36 & 5,67 & 32,15 \\
\hline ApSentimientos & 19,71 & 4,23 & 17,97 \\
\hline ApAcciones & 16,48 & 4,28 & 18,33 \\
\hline ApIdeas & 18,86 & 5,67 & 32,23 \\
\hline ApValores & 20,96 & 4,21 & 17,76 \\
\hline \multicolumn{4}{|c|}{ Facetas de la Amabilidad } \\
\hline AmConfianza & 16,80 & 4,40 & 19,37 \\
\hline AmFranqueza & 16,26 & 4,59 & 21,10 \\
\hline AmAltruismo & 21,69 & 4,15 & 17,27 \\
\hline AmActConcil & 16,30 & 4,50 & 20,33 \\
\hline AmModestia & 17,65 & 4,69 & 22,07 \\
\hline AmSensOtros & 19,94 & 3,56 & 12,72 \\
\hline \multicolumn{4}{|c|}{ Facetas de la Responsabilidad } \\
\hline RCompetencia & 20,11 & 3,83 & 14,69 \\
\hline ROrden & 17,75 & 4,09 & 16,74 \\
\hline RSentDeber & 21,34 & 3,97 & 15,83 \\
\hline
\end{tabular}




\section{Continuación Tabla 1}

\begin{tabular}{lccc}
\hline & Media & Desv. Típ. & Varianza \\
\hline Factores de la personalidad & & & \\
RNecLogro & 21,17 & 4,01 & 16,14 \\
RAutodisci & 19,30 & 5,07 & 25,78 \\
RDeliberación & 17,30 & 5,04 & 25,43 \\
Impulsividad & & & \\
BIS11 & 59,28 & 14,11 & 199,26 \\
Flexibilidad cognitiva & & & \\
TMTB & 94,40 & 40,50 & 1640,40 \\
WCSTAciertos & 63,60 & 18,82 & 354,30 \\
WCSTErroresPerseverativos & 13,63 & 12,38 & 153,26 \\
WCSTErroresNoPerseverativos & 20,42 & 12,29 & 151,22 \\
WCSTRespuestasConceptuales & 55,11 & 20,40 & 416,27 \\
WCSTCategorías & 4,56 & 1,55 & 2,41 \\
WCSTTiempototal & 1817,32 & 655,74 & 429998,46 \\
\hline
\end{tabular}

Fuente: Autores

Tabla 2. Coeficiente de correlaciones Spearman entre las variables de personalidad, flexibilidad cognitiva e impulsividad

\begin{tabular}{lcccccccc}
\hline & BIS & $(\mathbf{1})$ & $\mathbf{( 2 )}$ & $\mathbf{( 3 )}$ & $\mathbf{( 4 )}$ & $\mathbf{( 5 )}$ & $\mathbf{( 6 )}$ & $\mathbf{( 7 )}$ \\
\hline Neuro &, 03 &, 15 &,- 26 &, $43^{* *}$ &, 26 &,- 24 &,$- 37^{* *}$ &,- 11 \\
\hline Extra &,- 07 &,- 04 &,- 02 &, 03 &, 08 &,- 02 &,- 05 &,- 14 \\
\hline Aper &, 15 &, 01 &,- 14 &, 07 &,- 11 &,- 15 &,- 16 &,- 17 \\
\hline Ama &,$- 31^{*}$ &,- 25 &, 10 &,- 06 &,- 18 &, 10 &, 18 &, 03 \\
\hline Resp &,- 10 &,- 19 &, 15 &,- 17 &,- 07 &, 13 &, 06 &,- 11 \\
\hline NAns &,- 02 &, 08 &,- 15 &, 19 &, 16 &,- 13 &,- 22 &,- 12 \\
\hline NHos &, 20 &, 06 &,- 13 &, $27^{*}$ &, 11 &,- 14 &,$- 37^{* *}$ &,- 07 \\
\hline NDep &, 11 &, 12 &,- 21 &, $43^{* *}$ &, 12 &,- 21 &,$- 37^{*}$ &,- 03 \\
\hline NAnsSo &,- 01 &, 06 &,- 01 &, 12 &, $34^{*}$ &, 01 &,- 10 &, 26 \\
\hline NImp &, 03 &, 12 &,- 14 &, $29^{*}$ &, 10 &,- 11 &,- 19 &,- 19 \\
\hline NVul &,- 07 &, 16 &,$- 29^{*}$ &, $37^{* *}$ &, $28^{*}$ &,- 25 &,- 22 &,- 19 \\
\hline ECor &, 00 &,- 05 &,- 08 &, 01 &, 01 &,- 09 &,- 07 &,- 02 \\
\hline EGre &,- 06 &,- 14 &,- 01 &, 01 &, 19 &,- 01 &,- 07 &, 04 \\
\hline EAse &,- 03 &,- 03 &,- 09 &,- 03 &, 01 &,- 07 &,- 07 &,- 06 \\
\hline
\end{tabular}


Diferencias individuales en la impulsividad y la flexibilidad cognitiva en adultos jóvenes sanos

\begin{tabular}{|c|c|c|c|c|c|c|c|c|}
\hline EAct &,- 03 & , o3 &,- 00 & ,02 & ,13 & ,01 &,- 17 &,- 19 \\
\hline EBqEm &,- 16 & ,04 & ,09 & ,05 & , 05 & ,06 & ,09 &,- 23 \\
\hline EEmPo & ,07 &,- 07 &,- 02 &,- 05 &,- 02 &,- 02 &,- 03 &,- 21 \\
\hline ApFan &,- 05 & 16 &,- 19 & ,19 & ,05 &,- 22 &,- 14 &,- 13 \\
\hline ApEst &, $28^{*}$ &,- 01 &,- 21 & ,24 & ,01 &,- 19 &,- 20 &,- 16 \\
\hline ApSen & 22 & ,06 &,- 08 & , 07 &,- 04 &,- 11 &,- 11 &,- 21 \\
\hline ApAcc &,- 09 &,- 10 & ,o8 &,- 24 &,- 09 & ,07 & , 04 &,- 05 \\
\hline ApIde &, 21 &,- 05 &,- 02 &,- 03 &,- 02 &,- 02 &,- 05 &,- 05 \\
\hline ApVal &,- 02 & ,07 &,- 00 &,- 01 &,- 11 & ,01 & ,oo &,$- \mathrm{OO}$ \\
\hline AmCon & ,22 &,- 06 & ,01 & ,oo &,- 05 & ,02 & ,08 & ,04 \\
\hline AmFra &,$- 32^{*}$ &,$- 34^{*}$ & ,20 &,- 13 &,- 17 & ,22 &, 27 &,- 24 \\
\hline AmAlt &,- 07 &,- 01 &,- 09 & , o3 & ,10 &,- 04 & ,06 & ,03 \\
\hline AmAcCo &,- 18 &,- 08 & ,o8 &,- 08 &,- 14 & ,o8 & ,11 & ,23 \\
\hline AmMod &,- 24 &,$- 30^{*}$ & ,12 &,- 09 &,- 26 & , 09 & ,07 &,- 13 \\
\hline AmSeOt &,- 11 &,- 08 & ,oo & ,09 &,- 17 &,- 03 & , o8 & ,o6 \\
\hline RCom &,$- \mathrm{OO}$ &,- 18 & ,09 &,- 27 &,- 02 & ,13 & ,o8 &,$- 29^{*}$ \\
\hline ROrd & ,06 &,- 09 &,- 01 & ,06 & 17 &,- 00 & ,04 & ,08 \\
\hline RSeDe &,- 14 &,- 11 & ,06 & ,01 &,- 12 & ,05 & ,07 &,- 05 \\
\hline RNeLo &,- 19 &,- 09 & ,26 &,- 12 & , o3 & ,26 & ,02 &,- 04 \\
\hline RAut & , oo &,- 17 & ,17 &,- 23 &,- 19 & ,15 & ,15 &,- 07 \\
\hline RDel & ,09 &,- 15 & ,21 &,- 06 &,- 04 & ,18 & ,o8 & , O3 \\
\hline TMTB (1) & ,23 & 1,0 & & & & & & \\
\hline WCST/A (2) & ,07 &,$- 31^{*}$ & 1,0 & & & & & \\
\hline WCST/EP (3) & ,07 &, $36^{* *}$ & & 1,0 & & & & \\
\hline $\begin{array}{l}\text { WCST / EN P } \\
\text { (4) }\end{array}$ &,- 16 &, $57^{* *}$ & & & 1,0 & & & \\
\hline WCST/RC (5) & ,04 &,$- 29^{*}$ & & & & 1,0 & & \\
\hline WCST/C (6) &,- 08 &,$- 28^{*}$ & & & & & 1,0 & \\
\hline WCST/T (7) &,- 02 & $49^{* *}$ & & & & & & 1,0 \\
\hline
\end{tabular}

Fuente: Autores 


\section{Análisis y discusión}

El valor de la correlación entre la impulsividad y la amabilidad $(-, 307)$ refiere una asociación negativa que implica una tendencia a la disminución de los niveles de impulsividad en cuanto aumentan los niveles de amabilidad o a un aumento de los niveles de impulsividad en cuanto disminuyen los niveles de amabilidad. Las personas con altos niveles de amabilidad tienden a confiar en los demás, a ser francos, a sentir interés por los demás, a ser conciliadores y modestos. En general, este factor de la personalidad está caracterizado por una alta capacidad para controlar el comportamiento, lo cual repercute en una alta aceptabilidad social (Graziano, Jensen-Campbell \& Hair, 1996; Jensen-Campbell \& Graziano, 2001). Otra investigación (Robinson, 2007) reportó que la Amabilidad estaba asociada con un mayor control afectivo y emocional luego de que se presentaran pensamientos agresivos. También se ha encontrado, mediante análisis de correlaciones bivariadas, una asociación negativa (-0.16) y estadísticamente significativa $(p<.05)$ entre la Amabilidad y la capacidad para controlar el comportamiento (Vreeke \& Muris, 2012).

Las personas amables son percibidas como menos disruptivas en su comportamiento interpersonal (Moskowitz, 1994) y con una mayor habilidad para regularse a sí mismos (Ode \& Robinson, 2007; Ode, Robinson \& Wilkowski, 2007). Regularmente, las personas amables tienden a ser reflexivos y a considerar los costos y beneficios de los comportamientos sociales (Jensen-Campbell et al., 2002). El factor Amabilidad ha sido asociado con el proceso de control con esfuerzo, o auto regulación (Kochanska, Murray \& Coy, 1997), que se entiende como la habilidad para iniciar y detener voluntariamente la acción (Kochanska, Murray \& Harlan, 2000) o como la capacidad para suprimir un comportamiento dominante para ejecutar una respuesta no dominante (Jackson et al., 2010).

La Amabilidad también se ha asociado positivamente con la capacidad para inhibir respuestas. Se ha reportado (Jensen-Campbell et al., 2002) que este factor era el único de los cinco factores del NEO-PI que 
predecía el desempeño neuropsicológico en la prueba Stroop, de manera que los altos puntajes se asociaban con una mayor capacidad para controlar las respuestas, y en cuatro de las variables del WCST, de manera que los altos puntajes se asociaban con una mejor capacidad para modificar estrategias. Los análisis de correlación entre la Amabilidad y las variables del WCST de este estudio (tabla 2), aunque no arrojaron correlaciones estadísticamente significativas, los signos y valores de las correlaciones son congruentes con los resultados que asocian este factor con una mayor capacidad para modificar estrategias en el grado en que especifica el coeficiente. Este resultado coincide con la correlación entre este factor y el TMT-B. Si bien la correlación no fue estadísticamente significativa, el signo y el valor de la correlación indican que la Amabilidad está asociada con una mayor flexibilidad mental.

El factor Responsabilidad tuvo un comportamiento similar al de la Amabilidad en cuanto a las relaciones con la impulsividad y las variables neuropsicológicas. El valor de la correlación entre la Responsabilidad y la impulsividad no fue estadísticamente significativo pero el valor y el signo indican que los altos niveles del factor se asocian con menores niveles de impulsividad, en el grado en que especifica el coeficiente. La asociación con el TMT-B tuvo un comportamiento similar: el signo y el valor indican un mejor desempeño en la prueba cuanto mayor sean los niveles de Responsabilidad. De igual forma, las asociaciones con las variables del WCST indican que el aumento en los niveles del factor se asocia con un aumento en los niveles en la capacidad para modificar estrategias, en el grado en que especifica el coeficiente.

Las personas conscientes se caracterizan por ser ordenadas, sistemáticas, con alto sentido del deber, deliberativas, competentes y auto disciplinadas (McCrae y Costa, 2010). La Responsabilidad se contrapone directamente con los atributos cognitivos y comportamentales que caracterizan el concepto de "impulsividad". Las personas responsables deliberan sobre las acciones, tienen un comportamiento reflexivo y disponen de la capacidad para contener los actos aunque no exista una gratificación inmediata (Jackson et al., 2010). También se ha reporta- 
do que los bajos niveles de Responsabilidad se asocian con una menor capacidad para inhibir respuestas (Avisar \& Shalev, 2011). Sin embargo, los coeficientes de correlación de las facetas del factor (tabla 2) no respaldan completamente estas afirmaciones y hallazgos.

Si bien los resultados de este estudio no son concluyentes en cuanto a una asociación de "baja impulsividad" y "alta flexibilidad cognitiva" en los factores de Amabilidad y Responsabilidad, si puede apreciarse una tendencia hacia estas relaciones. Además, no es fortuito que sean estos dos factores, entre los cinco grandes, los que presenten esta tendencia. De acuerdo con los análisis de meta-rasgos del modelo de los cinco grandes factores (DeYoung, 2006; Digman, 1997), se ha logrado cierto consenso en relación con la existencia de dos grandes rasgos que reúnen los cinco factores. La Amabilidad y la Responsabilidad, junto con la Estabilidad Emocional, configuran el primer meta rasgo, denominado "Alfa", "Estabilidad", o "Auto control” se refiere, en general, a la estabilidad emocional, cognitiva y comportamental (DeYoung, 2010a).

Los factores de Amabilidad y Responsabilidad también se han asociado con el sistema serotoninérgico. La serotonina hace parte del sistema monoaminérgico y tiene efectos reguladores e inhibidores sobre el afecto, la cognición y el comportamiento (Spoont, 1992). Los bajos niveles de serotonina se han asociado con comportamientos agresivos y disruptivos caracterizados por una alta impulsividad y bajo control de los impulsos, como la violencia doméstica (George et al., 2001), la agresividad impulsiva (Cleare \& Bond, 1997) y los ataques físicos (Coccaro, Kavoussi, Cooper \& Hauger, 1997). Ambos factores de la personalidad se han asociado con altos niveles de serotonina, con lo cual es de esperar una baja impulsividad y un alto control emocional y cognitivo (Carver \& Miller, 2006). La serotonina se ha postulado como hipótesis bioquímica para explicar las diferencias en la capacidad de control inhibitorio y la flexibilidad cognitiva (Evers, van der Veen, Fekkes \& Jolles, 2007). 
Los resultados de la evaluación neuropsicológica en el factor Neuroticismo indican un bajo desempeño en la prueba del WCST. Los valores de las correlaciones entre el factor y las variables de "Errores perseverativos" y "Categorías" fueron estadísticamente significativas $(\mathrm{p}<.01)$ e indican una disminución en la capacidad para el razonamiento abstracto, la formación de conceptos y la generación de estrategias para afrontar contingencias a medida que aumenta el nivel de este factor de la personalidad. Las demás variables del WCST no presentaron correlaciones estadísticamente significativas pero los valores de los coeficientes son de consideración e indican la misma relación anterior.

Las facetas "Depresión” y "Hostilidad” fueron las que tuvieron las correlaciones más altas con las variables de "Errores perseverativos" y "Categorías”. La capacidad de resolución de problemas (WCST) se ha encontrado afectada en personas con depresión (Martin, Oren \& Boone, 1991). Ya se había reportado un aumento en las respuestas perseverativas en el WCST en personas con melancolía o depresión endógena (Austin, Mitchell \& Goodwin, 2001). En general, se ha reportado una afectación en el desempeño en tareas de función ejecutiva en personas con depresión (Koenigs \& Grafman, 2009). La hipótesis neuroanatómica-neuropsicológica sugiere que estos detrimentos cognitivos están asociados con un nivel de activación inadecuado de la corteza prefrontal dorsolateral. Esta corteza es el substrato de los procesos ejecutivos y su alteración funcional, en el caso de la depresión, conlleva a un deterioro en la capacidad cognitiva (Pietrzak, Spraguea \& Snyder, 2008).

La faceta "Impulsividad" no correlacionó con la variable de impulsividad del BIS-11. Este resultado podría explicarse por las características psicométricas de las pruebas. Tendría que revisarse la validez convergente y la validez de constructo de ambos instrumentos. Respecto a la relación entre la impulsividad (NEO) y la flexibilidad cognitiva, esta faceta obtuvo la tercera correlación positiva más alta con la variable del TMT-B. Si bien la correlación no fue estadísticamente significativa, sí se destaca el hecho 
de que haya sido positiva y superior a 1,0. Este valor refiere una tendencia a la disminución en la flexibilidad cognitiva con el aumento en los niveles de impulsividad en el grado en que especifica el coeficiente. No obstante, el grado de asociación es muy bajo como para poder aseverar algo concluyente. Pero las correlaciones con las variables del WCST confirman la presunción de que la impulsividad (NEO) está relacionada con un pobre desempeño en el razonamiento abstracto, la formación de conceptos y la generación de estrategias para afrontar contingencias.

Las relaciones entre la impulsividad (BIS) y varias medidas de funcionamiento ejecutivo se habían analizado en adultos jóvenes sanos y se encontró que los altos puntajes en impulsividad se asociaban con peores desempeños en tareas de planificación, control e inhibición (Koenigs \& Grafman, 2009). Si bien no evaluaron directamente la flexibilidad cognitiva, es de esperar, debido a la estructura factorial de la función ejecutiva, que la relación con la impulsividad también sea negativa. En otro análisis de la relación entre la impulsividad (BIS) y la función ejecutiva en adultos jóvenes sanos utilizando el TMT-B para evaluar la flexibilidad cognitiva se reportó una correlación parcial de 0,20 entre ambas variables (Keilpa, Sackeim \& Manna, 2005). Este dato coincide con el encontrado en el estudio (tabla 2).

El factor Extraversión no correlacionó significativamente con la impulsividad. Ya se había reportado una asociación entre la extraversión y la impulsividad, utilizando el Eysenck Personality Questionnaire (EPQ) (Eysenck \& Eysenck, 1978). Sin embargo, otro estudio (Campbell y Heller, 1987), utilizando el mismo instrumento, no reportó ningún tipo de asociación entre estas mismas variables. En esencia, no hay consenso respecto a si, teóricamente, la impulsividad debería aceptarse como una de las características de la extraversión (Whiteside \& Lynam, 2001). Hay posiciones a favor (Rocklin \& Revelle, 1981; Eysenck \& Eysenck, 1977) y en contra (Buss \& Plomin, 1975). Resulta difícil establecer un consenso entre los estudios que analizan la relación porque utilizan diferentes instrumentos para medir las dos variables. 
La Extraversión no correlacionó con ninguna de las medidas de flexibilidad cognitiva (tabla 2). La Apertura a la experiencia tampoco tuvo correlaciones con las variables de flexibilidad cognitiva, aunque las correlaciones con las variables del WCST podrían indicar cierta tendencia a la flexibilidad. Sin embargo, estadísticamente no hubo ninguna correlación significativa entre la Extraversión y la Apertura y las variables de flexibilidad cognitiva. Estos resultados no coinciden con los análisis teóricos y factoriales de los meta rasgos de la personalidad, según los cuales estos dos factores constituyen el factor beta o plasticidad que se caracteriza por la flexibilidad cognitiva (Avisar \& Shalev, 2011; DeYoung, Peterson \& Higgins, 2002).

\section{Conclusiones}

Solo el factor Amabilidad presentó una correlación estadísticamente significativa con la impulsividad. Este hallazgo no solo coincide con lo reportado en la literatura sobre neurociencia de la personalidad sino que es plenamente consistente con los atributos cognitivos que deberían estar asociados con el comportamiento que exhiben este tipo de personas. La correlación entre la Responsabilidad y la impulsividad, aunque no fue significativa, también coincide parcialmente con los reportes de la literatura y es consistente con la estructura del comportamiento de este tipo de personas.

Las correlaciones entre estos dos factores y las variables de flexibilidad también coinciden con las estructura de la personalidad correspondiente y se articulan adecuadamente con un modelo explicativo que incluye elementos neurobiológicos (DeYoung, 2010b). Las asociaciones con las variables de flexibilidad cognitiva también concuerdan con la estructura cognitiva y del comportamiento del Neuroticismo. En general, estos hallazgos coinciden con la teoría de los meta rasgos (DeYoung, Peterson \& Higgins, 2002), ya que estos tres factores de la personalidad comparten las características neuropsicológicas asociadas con el meta rasgo alfa. No se detectaron correlaciones entre los factores Extraversión y Apertura, y las variables de impulsividad y flexibilidad cognitiva. 


\section{Referencias}

Alcázar-Córcoles, M. A., Verdejo-García, A., Bouso-Saiz, J. C., Bezos-Saldaña, L. (2010). Neuropsychology of impulsive aggression. Revista de Neurología, 50(5), 291-299. Recuperado de http://www.ncbi.nlm.nih.gov/ pubmed/20217648.

Alcorn, J., Gowin, J., Green, C., Swann, A., Moeller, F., Lane, S. (2013). Aggression, impulsivity, and psychopathic traits in combined antisocial personality disorder and substance use disorder. The Journal of Neuropsychiatry and Clinical Neurosciences, 25, 229-232. doi:10.1176/appi.neuropsych.1203006o.

Austin, M. P., Mitchell, P., Goodwin, G. M. (2001). Cognitive deficits in depression: possible implications for functional neuropathology. The British Journal of Psychiatry, 178, 200-206. doi:10.1192/bjp.178.3.200.

Avisar, A., Shalev, L. (2011). Sustained attention and behavioral characteristics associated with ADHD in adults. Applied Neuropsychology, 18(2), 107-116. doi: 10.1080/09084282.2010.547777.

Barratt, E., Patton, J. H. (1983). Impulsivity: Cognitive, behavioral, and psychophysiological Correlates. En Zuckerman, M. (Ed.). The Biological basis of impulsivity and sensation seeking. New Jersey, USA: Lawrence Erlbaum Associates.

Berlin, H., Rolls, E., Iversen, S. (2005). Borderline Personality Disorder, Impulsivity, and the Orbitofrontal Cortex. American Journal of Psychiatry, 162, 12-18. doi:10.1176/appi.ajp.162.12.2360.

Buss, A., Plomin, R. (1975). A temperament theory of personality development. New York, USA: Wiley.

Campbell, J., Heller, J. F. (1987). Correlations of extraversion, impulsivity and sociability with sensation seeking and MBTI-introversion. Personality and Individual Differences, 8(1), 133-136. doi: 10.1016/0191-8869(87)90021-3.

Carver, C. S., Miller, C. J. (2006). Relations of serotonin function to personality: Current views and a key methodological issue. Psychiatry Research, 144, 1-15. doi: http://dx.doi.org/10.1016/j.psychres.2006.03.013. 
Chamberlain, S. R., Fineberg, N. A., Blackwell, A. D., Robbins, T. W., Sahakian, B. J. (2006). Motor inhibition and cognitive flexibility in obsessive-compulsive disorder and trichotillomania. The American Journal of Psychiatry, 163(7), 1282-1284. doi:10.1176/appi.ajp.163.7.1282.

Chamorro-Premuzic, T. (2011). Personality and Individual Differences. USA: John Wiley \& Sons.

Church, A. T., Katigbak, M. S. (2002). Indigenization of psychology in the Philippines. International Journal of Psychology, 37(3), 129-148. doi: 10.1080/00207590143000315.

Cleare, A. J., Bond, A. J. (1997). Does central serotonergic function correlate inversely with aggression? A study using D-fenfluramine in healthy subjects. Psychiatry Research, 69, 89-95. doi: 10.1016/So165-1781(96)03052-1.

Coccaro, E. F., Kavoussi, R. J., Cooper, T. B., Hauger, R. L. (1997). Central serotonin activity and aggression: Inverse relationship with prolactin response to D-fenfluramine, but not CSF 5-HIAA concentration, in human subjects. American Journal of Psychiatry, 154, 1430-1435. Recuperado de http://www.ncbi.nlm. nih.gov/pubmed/9326827.

Costa, P. T. Jr, McCrae, R. R. (1992). Revised NEO Personality Inventory (NEO PIR) and NEO Five Factor Inventory (NEO-FFI). Professional manual. Odessa, USA: Psychological Assessment Resources.

Cyders, M. A., Coskunpinar, A. (2011). Depression, impulsivity and health-related disability: A moderated mediation analysis. Journal of Research in Personality, 45(6), 679-682. doi: 10.1016/j.jrp.2011.08.005.

Dalley, J., Roiser, J. (2012). Dopamine, serotonin and impulsivity. Neuroscience, 215(26), 42-58. doi: 10.1016/j.neuroscience.2012.03.065.

DeYoung, C. G. (2006). Higher-order factors of the Big Five in a multi-informant sample. Journal of Personality and Social Psychology, 91, 1138-1151. doi: http://dx.doi.org/10.1037/o022-3514.91.6.1138. 
DeYoung, C. G. (2010a). Impulsivity as a personality trait. En Vohs, K., y Baumeister, R. (Eds.). Handbook of Self-Regulation: Research, Theory, and Applications (Second edition, pp. 485-502). New York, USA: Guilford Press.

DeYoung, C. G. (2010b). Personality neuroscience and the biology of traits. Social and Personality Psychology Compass, 4(12), 1165-1180. Disponible en http:// psych.wfu.edu/furr/255/DeYoung\%20(2010).pdf.

DeYoung, C. G., Peterson, J. B., Higgins, D. M. (2002). Higher-order factors of the Big Five predict conformity: Are there neuroses of health? Personality and Individual Differences, 33, 533-552. doi: 10.1016/j.jrp.2011.08.010.

DeYoung, C. G., Gray, J. R. (2009). Personality Neuroscience: Explaining Individual Differences in Affect, Behavior, and Cognition. En Corr, P. J., y Matthews, G. (Eds.), Cambridge handbook of personality psychology (pp. 323-346). New York, USA: Cambridge University Press.

Digman, J. M. (1997). Higher-order factors of the Big Five. Journal of Personality and Social Psychology, 73, 1246-1256. doi: http://dx.doi.org/10.1037/oo22$3514 \cdot 73 \cdot 6.1246$.

Eling, P., Derckx, K., Maes, R. (2008). On the historical and conceptual background of the Wisconsin Card Sorting Test. Brain and Cognition, 67, 247-253. doi: 10.1016/j.bandc.2008.01.006.

Evers, E. A., van der Veen, F. M., Fekkes, D., Jolles, J. (2007). Serotonin and cognitive flexibility: neuroimaging studies into the effect of acute tryptophan depletion in healthy volunteers. Current Medicinal Chemestry, 14(28), 2989-2995. doi: 10.2174/092986707782794032.

Eysenck, S. B. G., Eysenck, H. J. (1977). The place of impulsiveness in a dimensional system of personality description. British Journal of Social and Clinical Psychology, 16(1), 57-68. Recuperado de http://www.ncbi.nlm.nih.gov/pub$\operatorname{med} / 843784$. 
Eysenck, S., Eysenck, H. (1978). Impulsiveness and venturesomenss: Their position in a dimensional system of personality description. Psychological Reports, 43,1247-1255. doi: 10.2466/pro.1978.43.3f.1247.

Fischer, S., Smith, G. T., Anderson, K. G. (2003). Clarifying the role of impulsivity in bulimia nervosa. International Journal of Eating Disorders, 33(4), 406-411. doi: 10.1002/eat.10165.

Flehmig, H., Steinborn, M., Langner, R., Westhoff, K. (2007). Neuroticism and the mental noise hypothesis: Relationships to lapses of attention and slips of action in everyday life. Psychology Science, 49(4), 343-36o. Recuperado de https://www.researchgate.net/publication/26514554_Neuroticism_and_ the_mental_noise_hypothesis_Relationships_to_lapses_of_attention_and_ slips_of_action_in_everyday_life.

Fossati, A., Di Ceglie, A., Acquarini, E., Barratt, E. S. (2001). Psychometric properties of an Italian version of the Barratt Impulsiveness Scale-11 (BIS-11) in nonclinical subjects. Journal of Clinical Psychology, 57, 815-828. doi: 10.1002/ jclp.1051.

Galimberti, E., Martoni, R. M., Cavallini, M. C., Erzegovesi, S., Bellodi, L. (2012). Motor inhibition and cognitive flexibility in eating disorder subtypes. Progress in Neuro-Pharmacological and Biological Psychiatry, 36(2), 307-12. doi: 10.1016/j.pnpbp.2011.10.017.

George, D. T., Umhau, J. C., Phillips, M. J., Emmela, D., Ragan, P. W., Shoaf, S.E., Rawlings, R. R. (2001). Serotonin, testosterone, and alcohol in the etiology of domestic violence. Psychiatry Research, 104, 27-37. doi: 10.1016/So1651781(01)00292-X.

Graziano, W. G., Jensen-Campbell, L. A., Hair, E. C. (1996). Perceiving interpersonal conflict and reacting to it: The case for agreeableness. Journal of Personality and Social Psychology, 70, 820-835. Recuperado de http://www.ncbi.nlm. nih.gov/pubmed/8636901. 
Hartmann, A. S., Rief, W., Hilbert, A. (2001). Psychometric properties of the German version of the Barratt Impulsiveness Scale, Version 11 (BIS-11) for adolescents. Perceptual and Motor Skills, 112(2), 353-68. doi: 10.2466/o8.09.10. PMS.112.2.353-368.

Heaton, R. K., Chelune, G. J., Talley, J. L., Kay, G. G., Curtis, G. (1993). Wisconsin Card Sorting Test (WCST). Manual revised and expanded. Odessa, USA: Psychological Assessment Resources Inc.

Hinshaw, S. P. (2003). Impulsivity, emotion regulation, and developmental psychopathology: specificity versus generality of linkages. Annals of the New York Academy of Science, 1008, 149-59. doi: 10.1196/annals.1301.016.

Hur, Y., Bouchard, Jr. T. (1997). The Genetic Correlation Between Impulsivity and Sensation Seeking Traits. Behavior Genetics, 27, 455-463. Recuperado de http://www.ncbi.nlm.nih.gov/pubmed/9336082.

Jackson, J., Wood, D., Boggc, T., Waltond, K., Harmse, P., Robertsa, B. (2010). What Do Conscientious People Do? Development and Validation of the Behavioral Indicators of Conscientiousness. Journal of Research in Personality, 44(4), 501-511. doi: 10.1016/j.jrp.2010.06.005.

Jensen-Campbell, L. A., Rosselli, M., Workman, K., Santisi, M., Rios, J., Bojan, D. (2002). Agreeableness, conscientiousness, and effortful control processes. Journal of Research in Personality, 36, 476-489. doi: http://dx.doi. org/10.1016/Soo92-6566(02)00004-1.

Jensen-Campbell, L. A., Graziano, W. G. (2001). Agreeableness as a moderator of interpersonal conflict. Journal of Personality, 69, 323-362. doi: 10.1111/14676494.00148.

Karin, F., Helmers, K., Young, S., Pihl, R. (1997). Extraversion and behavioral impulsivity. Personality and Individual Differences, 23(3), 441-452. doi: 10.1016/ So191-8869(97)80010-4. 
Keilpa, J., Sackeim, H., Manna, J. (2005). Correlates of trait impulsiveness in performance measures and neuropsychological tests. Psychiatry Research, 135, 191-201. doi: 10.1016/j.psychres.2005.03.006.

Kelly, N. R., Bulik, C. M., Mazzeo, S. E. (2013). Executive functioning and behavioral impulsivity of young women who binge eat. The International Journal of Eating Disorders, 46(2), 127-139. doi: 10.1002/eat.22096.

Kenemans, J. L., Bekker, E. M., Lijffijt, M., Overtoom, C. C., Jonkman, L. M., Verbaten, M. N. (2005). Attention deficit and impulsivity: selecting, shifting, and stopping. International Journal of Psychophysiology . 58(1), 59-70. doi: 10.1016/j.ijpsycho.2005.03.009.

Kisa, C., Yildirim, S. G., Göka E. (2005). Impulsivity and mental disorders. Turkish Journal of Psychiatry, 16(1), 46-54. Recuperado de http://www.ncbi.nlm.nih. gov/pubmed/15793698.

Kochanska, G., Murray, K. T., Harlan, E. T. (2000). Effortful control in early childhood: continuity and change, antecedents, and implications for social development. Developmental Psychology, 36, 220-232. doi: http://dx.doi. org/10.1037/0012-1649.36.2.220.

Kochanska, G., Murray, K., Coy, K. C. (1997). Inhibitory control as a contributor to conscience in childhood: from toddler to early school age. Child Development, 68, 263-277. Recuperado de http://www.ncbi.nlm.nih.gov/pubmed/9180oo1.

Koenigs, M., Grafman, J. (2009). The functional neuroanatomy of depression:

Distinct roles for ventromedial and dorsolateral prefrontal cortex. Behavioral Brain Research, 201(2), 239-234. doi: 10.1016/j.bbr.2009.03.004.

Lassiter, G. H. (2009). Impulsivity: Causes, Control and Disorders. USA: Nova Science Publishers Inc.

Laursen, B., Pulkkinen, L., Adams, R. (2002). The antecedents and correlates of agreeableness in adulthood. Developmental Psychology, 38, 591-603. doi: 10.1037//0012-1649.38.4.591. 
Lawrence, A., Luty, J., Bogdan, N., Sahakian, B., Clark, L. (2009). Impulsivity and response inhibition in alcohol dependence and problem gambling. Psychopharmacology. 207(1), 163-172. doi: 10.1007/soo213-009-1645-x.

Lezak, M. D. (1995). Neuropsychological assessment (3rd ed.). New York, USA: Oxford University Press.

Logan, G., Schachar, R., Tannock, R. (1997). Impulsivity and Inhibitory Control. Psychological Science, 8(1), 60-64. Recuperado de http://pss.sagepub.com/ content/8/1/6o.abstract

Madden, G. J., Bickel, W. K. (2010). Impulsivity: The Behavioral and Neurological Science of Discounting. USA: American Psychological Association.

Mahoney, B. (2011). Personality and Individual Differences. Exeter, UK: Learning Matters Ltd.

Martin, D. J., Oren, Z., Boone, K. (1991). Major depressives' and dysthmics' performance on the Wisconsin Card Sorting Test. Journal of Clinical Psychology, 47(5), 684-90. Recuperado de http://www.ncbi.nlm.nih.gov/ pubmed/1939715.

McCrae, R. R., Costa, P. T. (1983). Joint factors in self-reports and ratings: Neuroticism, extraversion and openness to experience. Personality and Individual Differences, 4(3), 245-255. Recuperado de http://www.sciencedirect.com/ science/article/pii/o191886983901460.

McCrae, R. R., Costa, P. T. (2010). NEO Inventorie: professional manual. Lutz, USA: Psychological Assessment Resources, Inc.

Moore, A., Malinowski, P. (2009). Meditation, mindfulness and cognitive flexibility. Consciousness and Cognition, 18(1), 176-186. doi: 10.1016/j.concog.2008.12.008.

Moskowitz, D. S. (1994). Cross-situational generality and the interpersonal circumplex. Journal of Personality and Social Psychology, 66, 921-933. Recuperado de http://psycnet.apa.org/index.cfm?fa=buy.optionToBuy\&id=1994-37308-001. 
Ode, S., Robinson, M., Wilkowski, B. (2007). Can One’s Temper be Cooled? A Role for Agreeableness in Moderating Neuroticism's Influence on Anger and Aggression. Journal of Research in Personality, 42(2), 295-311. doi: 10.1016/j. jrp.2007.05.007.

Ode, S., Robinson M. (2007). Agreeableness and the Self-Regulation of Negative Affect: Findings Involving the Neuroticism/Somatic Distress Relationship. Personality and Individual Differences, 43(8), 213-2148. doi: 10.1016/j. paid.2007.06.035.

Perugi, G., Del Carlo, A., Benvenuti, M., Fornaro, M., Toni, C., Akiskal, K., Akiskal, H. (2011). Impulsivity in anxiety disorder patients: Is it related to comorbid cyclothymia? Journal of Affective Disorders, 133(3), 600-606. doi: 10.1016/j. jad.2011.04.033.

Pickering, A. D. (2004). The neuropsychology of impulsive antisocial sensation seeking personality traits: From dopamine to hippocampal function? En Stelmack, R. M. (Ed.), On the psychobiology of personality: Essays in honor of Marvin Zuckerman (pp. 453-477). New York, USA: Elsevier.

Pietrzak, H., Spraguea, A., Snyder, P. (2008). Trait impulsiveness and executive function in healthy young adults. Journal of Research in Personality, 42, 13471351. doi: 10.1016/j.jrp.2008.03.004.

Plomin, R. (1976). Extraversion: sociability and impulsivity? Journal of Personality Assessment, 4O(1), 24-30. Recuperado de http://www.ncbi.nlm.nih.gov/pub$\operatorname{med} / 1255395$.

Reitan, R. M. (1959). Manual for administration of neuropsychological test batteries for adults and children. Unpublished manuscript.

Ritter, S., Damian, R., Simonton, D., van Baaren, R., Strick, M., Derks, J., Dijksterhuis, A. (2012). Diversifying experiences enhance cognitive flexibility. Journal of Experimental Social Psychology, 48, 961-964. doi: 10.1016/j. jesp.2012.02.00. 
Roberts, W., Fillmore, M. T., Milich, R. (2011). Linking impulsivity and inhibitory control using manual and oculomotor response inhibition tasks. Acta Psychologica, 138(3), 419-428. 2011 doi:10.1016/j.actpsy.2011.09.002.

Robinson, M. D. (2007). Personality, Affective Processing, and Self-Regulation: Toward Process-Based Views of Extraversion, Neuroticism, and Agreeableness. Social and Personality Psychology Compass, 1(1), 223-235. doi: 10.1111/j.1751-9004.2007.00019.x.

Rocklin, T., Revelle, W. (1981). The measurement of extraversion: A comparison of the Eysenck Personality Inventory and the Eysenck Personality Questionnaire. British Journal of Social Psychology, 20(4), 279-284. Recuperado de http:// www.personality-project.org/revelle/publications/rr81.small.pdf.

Semenova, S. (2012). Attention, impulsivity, and cognitive flexibility in adult male rats exposed to ethanol binge during adolescence as measured in the five-choice serial reaction time task: the effects of task and ethanol challenges. Psychopharmacology, 219(2), 433-434. doi: 10.1007/s00213-011-2458-2.

Spoont, M. R. (1992). Modulatory role of serotonin in neural information processing: Implications for human psychopathology. Psychological Bulletin, 112, 330350. doi: http://dx.doi.org/10.1037/0033-2909.112.2.330.

Squillace, M., Picón, J., Schmidt V. (2011). El concepto de impulsividad y su ubicación en las teorías psicobiológicas de la personalidad. Revista de Neuropsicologia Latinoamericana, 3(1), 8-18. Recuperado de http://pepsic.bvsalud.org/ pdf/rnl/v3n1/v3n1ao2.pdf.

Strack, F., Deutsch, R. (2004). Reflective and Impulsive Determinants of Social Behavior. Personality and Social Psychology Review, 8(3), 220-24. doi: 10.1207/ S15327957pspro803_1.

Su, Y-F., Chung, S. H., Su, S. W. (2012). The Impact of Cognitive Flexibility on Resistance to Organizational Change. Social Behavior and Personality: an International Journal, 4O(5). 
Swann, A., Steinberg, J., Lijffijt, M., Moeller, F. (2008). Impulsivity: Differential relationship to depression and mania in bipolar disorder. Journal of Affective Disorders, 106(3), 241-248. doi: http://dx.doi.org/10.1016/j.jad.2007.07.011.

Tombaugh, T. N. (2004). Trail Making Test A and B: Normative data stratified by age and education. Archives of Clinical Neuropsychology, 19(2), 203-214. doi: 10.1016/So887-6177(03)ooo39-8.

Torregrossa, M., Quinnm J., Taylor J. (2008). Impulsivity, Compulsivity, and Habit: The Role of Orbitofrontal Cortex Revisited. Biological Psychiatry, 63(3), 253255. doi: 10.1016/j.biopsych.2007.11.014.

Vaz-Leal, F. L., Rodríguez-Santos, L., García-Herráiz, M. A., Chimpén-López, C. A., Rojo-Moreno, L., Beato-Fernández, L., Ramos-Fuentes, M. I. (2014). Papel de la depresión y la impulsividad en la psicopatología de la bulimia nerviosa. Revista de Psiquiatría y Salud Mental, 7(1), 25-31. doi: 10.1016/j. rpsm.2013.06.003.

Votruba, K. L., Rapport, L. J., Vangel, S. J., Hanks, R. A., Lequerica, A., Whitman, R. D., Langenecker, S. (2008). Impulsivity and traumatic brain injury: the relations among behavioral observation, performance measures, and rating scales. The Journal of Head Trauma Rehabilitation, 23(2), 65-73. doi: 10.1097/01. HTR.oooo314525.93381.69.

Vreeke, L. J., Muris, P. (2012). Relations Between Behavioral Inhibition, Big Five Personality Factors, and Anxiety Disorder Symptoms in Non-Clinical and Clinically Anxious Children. Child Psychiatry and Human Development, 43(6), 884-894. doi: 10.1007/s10578-012-0302-5.

Whiteside, S., Lynam, D. (2001). The Five Factor Model and impulsivity: using a structural model of personality to understand impulsivity. Personality and Individual Differences, 30, 669-689. doi: 10.1016/So191-8869(oo)ooo64-7.

Wing, V. C., Rabin, R. A., Wass, C. E., George, T. P. (2013). Correlations between executive function, decision-making and impulsivity are disrupted in schizo- 
phrenia versus controls. Psychiatry Research, 205(1-2), 168-171. doi: 10.1016/j.psychres.2012.08.022.

Yurgelun-Todd, D. A., Bueler, C. E., McGlade, E. C, Churchwell, J. C., Brenner, L. A., Lopez-Larson, M. P. (2011). Neuroimaging correlates of traumatic brain injury and suicidal behavior. The Journal of Head Trauma Rehabilitation, 26(4), 276-289. doi: 10.1097/HTR.obo13e31822251dc.

Zern, D. S., Kenney, H. J., Kvaraceus, W. C. (1977). The cognitive style dimension of reflectivity-impulsivity in normal and emotionally disturbed adolescents. The Journal of Genetic Psychology, 131(1st Half), 107-113. Recuperado de http:// www.ncbi.nlm.nih.gov/pubmed/915490. 